\title{
On Theorems of Jackson and Bernstein Type in the Complex Plane
}

\author{
J. M. Anderson, A. Hinkkanen, and F. D. Lesley
}

\begin{abstract}
We consider best polynomial approximation to functions analytic in a Jordan domain $D$ and continuous on $\bar{D}$. We relate theorems of Jackson and Bernstein type to the Hölder continuity of the exterior conformal mapping functions for $D$.
\end{abstract}

\section{Introduction}

Two of the fundamental theorems in the "constructive theory" of approximation by complex polynomials are those of Jackson and Bernstein. We state them in the very simple case that the domain in question is the unit disk $\Delta=\{|z|<1\}$. We write $f \in \operatorname{Lip}(\alpha)$ on a set $S$ to mean that $f$ is uniformly Hölder continuous on $S$ with exponent $\alpha$ : there exists a constant $K$ such that for all $z_{1}, z_{2} \in S$

$$
\left|f\left(z_{1}\right)-f\left(z_{2}\right)\right| \leq K\left|z_{1}-z_{2}\right|^{\alpha} .
$$

For a given set $S$ we write $\|g\|_{S}=\sup \{|g(z)|, z \in S\}$. The order of best approximation to $f$ on $S$ is $E_{n}(f)=\inf \left\{\left\|f-P_{n}\right\|_{S}, P_{n}\right.$ a polynomial of degree $\leq n$ in $\left.z\right\}$. Then Jackson's theorem for the disk is

$$
\begin{aligned}
& \text { If } f \text { is analytic in } \Delta \text { and } \operatorname{Lip}(\alpha) \text { on } \bar{\Delta} \text { for } \alpha \in(0,1], \\
& \text { then } E_{n}(f)=O\left(1 / n^{\alpha}\right) \text { on } \bar{\Delta} .
\end{aligned}
$$

Bernstein's theorem in this context is

$$
\text { For } \alpha \in(0,1) \text {, if } E_{n}(f)=O\left(1 / n^{\alpha}\right) \text { on } \bar{\Delta} \text {, then } f \in \operatorname{Lip}(\alpha) \text { on } \bar{\Delta} \text {. }
$$

This paper is addressed to the question: for which Jordan domains $D$ are these theorems true?

The answer to this question depends on the behavior of the exterior conformal mapping functions for $D$. Let $\Delta$ and $\Delta^{*}$ be respectively the interior and exterior of the unit circle in the $w$ plane. We denote the boundary $\partial D$ of $D$ by $C$ and

Date received: June 3, 1987. Date Revised: August 26, 1987. Communicated by Dieter Gaier. AMS classification: $30 \mathrm{E} 10,41 \mathrm{A10}, 41 \mathrm{A1} 17,30 \mathrm{C} 10$.

Key words and phrases: Jackson's theorem, Bernstein's theorem, Bernstein's inequality, Hölder continuity. 
denote by $D^{*}$ the exterior Ext $C$ of $C$. For $w \in \Delta^{*}$, we let

$$
\Phi(w)=a_{-1} w+\sum_{n=0}^{\infty} a_{n} w^{-n}
$$

be a conformal mapping of $\Delta^{*}$ onto $D^{*}$, and we let, $\Phi^{-1}=\Psi$. If $C$ is a curve of bounded rotation, then a result of Kövari [7] implies that if $\Phi \in \operatorname{Lip}(\beta)$ on $\bar{\Delta}^{*}$ and $f \in \operatorname{Lip}(\alpha)$ on $\bar{D}$ for $\alpha \leq 1$ and $\beta \leq 1$, then $E_{n}(f)=O\left(1 / n^{\alpha \beta}\right)$. Theorems of Jackson type have been considered by Belyi [4], the results being expressed in terms of the quantity $\rho(1+1 / n, z)$ which is the distance from the point $z \in C$ to the level line $\{\Phi(w):|w|=1+1 / n\}$. In particular, Belyi's results imply that if $C$ is a rectifiable quasicircle and if $\Phi \in \mathrm{Lip}(1)$ on $\bar{\Delta}^{*}$, then Jackson's theorem is true on $\bar{D}$. (See also pp. 54-56 of [6].) Further results of this type have been obtained by Andreevski [3]. We remark that $\Phi \in \operatorname{Lip}(1)$ if $C$ has a continuously turning tangent (i.e., $C$ is smooth), and if, in addition, the tangent angle has modulus of continuity as a function of arclength which satisfies a Dini condition. Smoothness alone is neither necessary nor sufficient for $\Phi \in \operatorname{Lip}(1)$ (see [9] and [11]).

Converse theorems, expressed again in terms of $\rho(1+1 / n, z)$ hold for arbitrary compact connected sets, as was proved by Lebedev and Tamrazov [8]. (The direct theorem is not true in this amount of generality, as was shown by Shirokov [14].) In our case, the result of $\left[8\right.$, p. 1358] shows that if $\Psi \in \operatorname{Lip}(1)$ on $\bar{D}^{*}$, then Bernstein's theorem is true on $\bar{D}$. This leads naturally to the conjectures, already partially established:

(1.1) Jackson's theorem holds on $\bar{D}$ if and only if $\Phi \in \operatorname{Lip}(1)$ on $\bar{\Delta}^{*}$.

(1.2) Bernstein's theorem holds on $\bar{D}$ if and only if $\Psi \in \operatorname{Lip}(1)$ on $\bar{D}^{*}$.

Note that our conjectures do not require that $C$ be a quasicircle, so that the sufficiency of the condition for $(\mathrm{J})$ is not established by the results of Belyi and Andreevski. Nor do we address that problem here. We are principally concerned with the necessity of the conditions for (B) and (J). In Section 2 of this paper we use a method of Szegö [15] to prove local generalizations of Bernstein's inequality, on which the proof of Bernstein's theorem is based. This celebrated inequality, stated for the disk $\bar{\Delta}$ is: let $P_{n}$ be a polynomial of degree $n$ with $\left\|P_{n}\right\|_{\bar{\Delta}} \leq 1$. Then $\left\|P_{n}^{\prime}\right\|_{\bar{\Delta}} \leq n$. We will then use these generalizations, which depend only on the local behavior of $\Psi$ at a boundary point, to prove new versions of Bernstein's theorem. One of these is a new proof that $\Psi \in \operatorname{Lip}(1)$ on $\bar{D}^{*}$ implies Bernstein's theorem. Another will imply that if $\Phi$ fails to be Lip(1) at a single point of $C$ in a very weak sense, then Jackson's theorem cannot be true.

In Section 3 we apply the method of strip mappings to study the local boundary behavior of the conformal mappings. First we prove a theorem, of independent interest we believe, that allows us to infer behavior of the mapping function for unrestricted approach to a boundary point from behavior on a "relatively thick" sequence. This result then strengthens our necessary condition for $(J)$ to hold. Then we use a strip mapping to construct a class of examples which are used in examining the necessity of the condition for Bernstein's theorem.

In Section 4 we then use these examples, together with the methods of [2], to demonstrate that if $\Psi$ fails to be $\operatorname{Lip}(1)$ at a single point of $C$, in a weak sense, 
then Bernstein's theorem may fail to be true, so that the condition is "almost necessary."

\section{Bernstein's Inequalities and Applications}

We begin by proving a local generalization of the classical Bernstein inequality. The method of proof is adapted from that of Szegö [15], who was concerned with domains $D$ which are bounded by piecewise analytic arcs meeting at angles. Our hypothesis requires only that $\partial D=C$ be a Jordan curve and that the function $\Psi$ be in $\operatorname{Lip}(1 / \beta)$ for $\beta \in(0,2]$, at the point in question (say $z=1$ ). There have been many geometric criteria given for such continuity in recent years [10], [12]. A particularly simple one is that the domain $D$ be bounded by a quasicircle and contain a wedge of opening $(2-\beta) \pi$ with vertex at $z=1$. Szegö's method is based on the following observation. The exterior mapping function $\Psi$ is conformal at $\infty$, with $\Psi(\infty)=\infty$. Thus, if $P_{n}$ is a polynomial of degree $n$, the function $P_{n}(z) / \Psi^{n}(z)$ is analytic at $\infty$. Furthermore, if $\left|P_{n}(z)\right| \leq 1$ on $\bar{D}$, then the maximum principle guarantees that

$$
\left|P_{n}(z)\right| \leq\left|\Psi^{n}(z)\right| \quad \text { for } \quad z \in \bar{D}^{*} .
$$

Theorem 1. Suppose that $C$ is a Jordan curve with interior $D$ and exterior $D^{*}$, and that $z=1 \in C$. Suppose that $\Psi$ maps $D^{*}$ conformally onto $\Delta^{*}$ and that there exist $K>0$ and $\beta \in(0,2]$ such that, for $z \in \bar{D}^{*}$ with $|z-1| \leq 1$,

$$
|\Psi(z)-1| \leq K|z-1|^{1 / \beta} \text {. }
$$

Then for any polynomial $P_{n}$ of degree $n$, for which $\left\|P_{n}\right\|_{\bar{D}} \leq 1$, we have

$$
\left|P_{n}^{\prime}(1)\right|<e^{K} n^{\beta} \text {. }
$$

Further, for $|z-1| \leq 1 /\left(2 n^{\beta}\right)$,

$$
\left|P_{n}(z)-P_{n}(1)\right|<4 e^{K} n^{\beta}|z-1| .
$$

Proof. By Cauchy's formula, for $\rho>0$, we have

$$
\left|P_{n}^{\prime}(1)\right| \leq(1 / \rho)\left(\max _{|z-1|=\rho}\left|P_{n}(z)\right|\right) .
$$

On account of (2.1), for $z \in \bar{D}^{*}$ and $|z-1|=\rho$,

$$
\begin{aligned}
\left|P_{n}(z)\right| & \leq|\Psi(z)|^{n}=|1+(\Psi(z)-1)|^{n} \\
& \leq(1+|\Psi(z)-1|)^{n} \\
& \leq\left(1+K \rho^{1 / \beta}\right)^{n}=\left(1+\left(K n \rho^{1 / \beta}\right) / n\right)^{n} .
\end{aligned}
$$

Now choose $\rho=n^{-\beta}$ so that, for $|z-1|=\rho$,

$$
\left|P_{n}(z)\right| \leq(1+K / n)^{n}<e^{K},
$$

and (2.3) follows from (2.5). 
For $|\zeta-1|<\rho / 2$ we have

$$
\left|P_{n}^{\prime}(\zeta)\right|<(4 / \rho) e^{K} \leq 4 n^{\beta} e^{K},
$$

from which (2.4) follows by integration.

Corollary 1. Suppose that $D$ is a Jordan domain, and that the exterior mapping function $\Psi \in \operatorname{Lip}(1)$ on $\bar{D}^{*}$. Then Bernstein's theorem is true on $\bar{D}$.

Proof. For $\beta=1$, the above Lemma is essentially the classical Bernstein inequality, from which Bernstein's theorem for $\bar{D}$ follows as in $[5$, p. 200].

Our next result assumes that the difference quotient associated with Hölder continuity converges to 0 for uniform approach in $\bar{D}^{*}$. The result will be used to establish a strengthening of Bernstein's theorem.

Theorem 2. Suppose that for $\Psi$ as above there exists a function $g$, continuous and increasing for $t \geq 0$, with $g(0)=0, g(1)>1$, and such that, for some $\beta \in(0,2]$,

$$
|\Psi(z)-1| \leq|z-1|^{1 / \beta} g(|z-1|)
$$

for all $z \in \bar{D}^{*}$ with $|z-1| \leq 1$. Then there exists a function $h(n)$ defined for $n=0$, $1,2, \ldots$, with $h(n) \rightarrow \infty$ monotonically as $n \rightarrow \infty$, such that, for any polynomial of degree $n$ with $\left\|P_{n}\right\|_{\bar{D}} \leq 1$, we have

$$
\left|P_{n}^{\prime}(1)\right|<e n^{\beta} / h(n) \text {. }
$$

Further, for $|z-1| \leq h(n) /\left(2 n^{\beta}\right)$, we have

$$
\left|P_{n}(z)-P_{n}(1)\right|<\left(4 n^{\beta} / h(n)\right)|z-1| \text {. }
$$

Proof. As in the proof of Theorem 1, for $|z-1|=\rho \leq 1$, we have

$$
\begin{aligned}
\left|P_{n}(z)\right| & \leq|\Psi(z)|^{n} \leq(1+|\Psi(z)-1|)^{n} \\
& \leq\left(1+n \rho^{1 / \beta} g(\rho) / n\right)^{n} .
\end{aligned}
$$

For each $n \geq 1$ let $h(n)$ be the unique solution to the equation $g\left(x / n^{\beta}\right)=1 / x^{1 / \beta}$, so that

$$
g\left(h(n) / n^{\beta}\right)=1 / h^{1 / \beta}(n) .
$$

Since $g(1)>1$, we have $h(1)<1$. Set $h(0)=h(1) / 2$. Because $g$ is increasing, $h(n) \rightarrow \infty$ monotonically, so that $1 / h^{1 / \beta}(n)$ decreases to 0 . By (2.9) it follows that $h(n) / n^{\beta}$ decreases to 0 . Thus, for $|z-1|=\rho=h(n) / n^{\beta}$,$$
\begin{aligned}
\left|P_{n}(z)\right| & \leq\left(1+h^{1 / \beta}(n) g\left(h(n) / n^{\beta}\right) / n\right)^{n} \\
& =(1+1 / n)^{n}<e,
\end{aligned}
$$

and, by (2.5),

$$
\left|P_{n}^{\prime}(1)\right|<e n^{\beta} / h(n)
$$


so that (2.7) is established. The inequality (2.8) then follows as in the proof of Theorem 1.

We now apply the above extension of Bernstein's inequality to prove a local theorem of Bernstein type. The case $\beta=1$ will then be applied to obtain a necessary condition for the Jackson property. In the rest of the paper \|| $\mid$ will denote \|\|$_{\vec{D}}$.

Theorem 3. Let $D$ be a Jordan domain with $1 \in C=\partial D$, and such that there exists a function $g$ as in Theorem 2 such that if $\Psi$ is the exterior mapping function for $D$, we have

$$
|\Psi(z)-1| \leq|z-1|^{1 / \beta} g(|z-1|)
$$

for fixed $\beta \in(0,2]$ and all $z \in \vec{D}_{*}^{*}$ with $|z-1| \leq 1$. Suppose that $f$ is analytic in $D$ and continuous on $\bar{D}$ with $E_{n}(f)=O\left(1 / n^{\alpha \beta}\right)$ for some $\alpha \in(0,1)$. Then, for $z \in \bar{D}$,

$$
|f(z)-f(1)| /|z-1|^{\alpha} \rightarrow 0 \quad \text { as } \quad z \rightarrow 1 .
$$

Proof. Let $h(n)$ be the function for which (2.7) holds as in Theorem 2. Let $\left\{P_{n}\right\}$ be a sequence of polynomials, $P_{n}$ of degree $\leq n$, such that $\left\|f-P_{n}\right\|=O\left(1 / n^{\alpha \beta}\right)$. Now let $V_{0}=P_{1}$ and let $V_{n}=P_{2^{n}}-P_{2^{n-1}}$, so that $f=\sum V_{n}$. Then, for $m \geq 1$, we have

$$
\begin{aligned}
|f(z)-f(1)| & \leq \sum_{0}^{m}\left|V_{n}(z)-V_{n}(1)\right|+\sum_{m+1}^{\infty}\left|V_{n}(z)-V_{n}(1)\right| \\
& \leq 4 \sum_{0}^{m}\left(2^{n \beta} / h\left(2^{n}\right)\right)\left\|V_{n}\right\||z-1|+2 \sum_{m+1}^{\infty}\left\|V_{n}\right\|,
\end{aligned}
$$

assuming that (2.8) holds for $n \leq 2^{m}$.

Now, for $n \geq 1$,

$$
\left\|V_{n}\right\| \leq\left\|P_{2^{n}}-f\right\|+\left\|P_{2^{n-1}}-f\right\| \leq K_{1} 2^{-n \alpha \beta},
$$

so that

$$
|f(z)-f(1)| \leq 4 K_{1}|z-1| \sum_{0}^{m}\left(2^{n \beta(1-\alpha)} / h\left(2^{n}\right)\right)+2 K_{1} \sum_{m+1}^{\infty} 2^{-n \alpha \beta} .
$$

We now define

$$
\varepsilon_{m}=2\left(\sum_{0}^{m} 2^{n \beta(1-\alpha)} / h\left(2^{n}\right)\right) /\left(\sum_{0}^{m} 2^{n \beta(1-\alpha)}\right)
$$

and note that $\varepsilon_{m} \rightarrow 0$ as $m \rightarrow \infty$. We then consider the sequence

$$
\begin{aligned}
\delta_{m} & =1 /\left(2^{m \beta} \varepsilon_{m}\right)=\left(\sum_{n=0}^{m} 2^{n \beta(1-\alpha)}\right) /\left(2^{m \beta}\left(2 \sum_{n=0}^{m} 2^{n \beta(1-\alpha)} / h\left(2^{n}\right)\right)\right) \\
& <\left(\sum_{n=0}^{m} 2^{n \beta(1-\alpha)}\right) /\left(2^{m \beta}\left(2 \sum_{n=0}^{m} 2^{n \beta(1-\alpha)} / h\left(2^{m}\right)\right)\right)=h\left(2^{m}\right) /\left(2 \times 2^{m \beta}\right) .
\end{aligned}
$$

Since $h(n) / n^{\beta}$ decreases to 0 as $n \rightarrow \infty$, it follows that $\delta_{m} \rightarrow 0$ as $m \rightarrow \infty$. 
Now suppose that $|z-1|=\delta$ for $z \in D$, and choose $m$ so that $\delta_{m+1} \leq \delta<\delta_{m}$. Then (2.8) holds for $n \leq 2^{m}$, and, for constants $K_{j}$ independent of $m$,

$$
\begin{aligned}
|f(z)-f(1)| & \leq K_{2} \delta \varepsilon_{m} 2^{m \beta(1-\alpha)}+K_{3} 2^{-m \beta \alpha} \\
& \leq \delta^{\alpha}\left(K_{2} \varepsilon_{m}\left(2^{m \beta} \delta_{m}\right)^{(1-\alpha)}+K_{4}\left(2^{(m+1) \beta} \delta_{m+1}\right)^{-\alpha}\right) \\
& \leq \delta^{\alpha}\left(K_{2} \varepsilon_{m}\left(1 / \varepsilon_{m}\right)^{(1-\alpha)}+K_{4}\left(\varepsilon_{m+1}\right)^{\alpha}\right) \\
& =\delta^{\alpha}\left(K_{2} \varepsilon_{m}^{\alpha}+K_{4} \varepsilon_{m+1}^{\alpha}\right)
\end{aligned}
$$

and the theorem is established.

Corollary 2. Suppose that $D$ is a Jordan domain with $1 \in C=\partial D$ and that $\Phi$ is a conformal mapping of $\{|w|>1\}$ onto $D^{*}=$ Ext $C$. Suppose that $\Phi$ fails to be in $\mathrm{Lip}(1)$ at the point $w=1$ in the sense that (2.10) holds, with $\beta=1$. Then Jackson's theorem cannot hold for $\bar{D}$.

Proof. Let $f(z)=1+(z-1)^{\alpha}$ in $\bar{D}$, for any $\alpha \in(0,1)$. If Jackson's theorem held then $E_{n}(f)=O\left(1 / n^{\alpha}\right)$. But then, by Theorem 3, (2.11) must hold. This being false for the given function, the corollary is proved.

\section{Some Results on Local Boundary Behavior}

There are many criteria which guarantee that (2.10) holds, and that Jackson's theorem then fails. That $D$ contain an angle with vertex at $z=1$, of opening larger than $\pi$, is one such criterion [12]. However, our conjecture is that $\Phi \in \operatorname{Lip}(1)$ on $\bar{\Delta}^{*}$ is necessary for Jackson's theorem and our Theorem 3 falls short of proving this, because the mapping function $\Phi$ may fail to be in $\operatorname{Lip}(1)$ on $\bar{\Delta}^{*}$, but there may be no point at which (2.10) holds. An example of this phenomenon may be constructed using the techniques of [11]. However, we can strengthen our Theorem 3 by proving the following result, which guarantees local continuity for arbitrary approach to a boundary point (as in (2.2) or (2.10)) from behavior on a suitably thick sequence.

Theorem 4. Suppose that $C=\partial D$ is a quasicircle and that $\Psi$ is the conformal mapping of $D^{*}=$ Ext $C$ onto $\{|w|>1\}$, with $\Psi(\infty)=\infty$ and $\Psi(1)=1$. Suppose that $\left\{z_{n}^{*}\right\} \subset \bar{D}^{*}$ is a sequence converging to 1 and that, for some positive constant $K$,

$$
\frac{\left|z_{n+1}^{*}-1\right|}{\left|z_{n}^{*}-1\right|} \geq K \frac{\left|\Psi\left(z_{n}^{*}\right)-1\right|}{\left|z_{n}^{*}-1\right|} \rightarrow 0 \text {. }
$$

Then there exists $g$ monotonic and continuous with $g(0)=0$ such that, for $z \in \bar{D}^{*}$ with $|z-1| \leq 1$,

$$
|\Psi(z)-1| \leq|z-1| g(|z-1|) .
$$

As in [9]-[12], it suffices to prove a corresponding result for strip mappings where the strip domains are the images of $D^{*}$ and $\Delta^{*}$ under a logarithmic transformation. In particular, if we assume that $z=-1 \in C$, then the mapping $w=T(z)=\log ((z+1) /(z-1))$ will take $D^{*}$ onto a strip domain $S$ which is 
bounded by two Jordan arcs, which we will call $C_{1}$ and $C_{2}$, each with $-\infty$ and $+\infty$ as endpoints: each $C_{j}$ can be parametrized as $u_{j}(x)+i v_{j}(x), x \in(-\infty, \infty)$, with $u_{j}(x) \rightarrow \pm \infty$ as $x \rightarrow \pm \infty$. The fact that the original curve $C$ is a quasicircle implies the existence of a positive constant $M$ with the following properties.

(3.2) Suppose that $w=u+i v \in \bar{S}$ and that $u^{\prime}-u \geq M$. Let $\sigma_{u^{\prime}}$ be the Ahlfors crosscut of $S$ at $u^{\prime}$ (see p. 349 of [10]). Then $\sigma_{u^{\prime}}$ separates $w$ from $+\infty$.

(3.3) Suppose that $w=u+i v \in \bar{S}$ and that $u-u^{\prime} \geq M$. Then $w$ is in the component of $\bar{S}-\sigma_{u^{\prime}}$ with $+\infty$ as a boundary point.

If $\theta(t)$ is the length of $\sigma_{t}$, then $\theta(t)<2 \pi$ for all $t$.

For the proofs of these properties, see [10].

Theorem 5. Let $\Sigma=\{z=x+i y:|y|<\pi / 2\}$ and let $S$ be a simply connected strip domain in the $w=u+i v$ plane, which is bounded by two Jordan arcs $C_{1}$ and $C_{2}$, each with $-\infty$ and $+\infty$ as endpoints. Further, let $S$ have the properties (3.2), (3.3), and (3.4). Let $F$ be a conformal mapping of $S$ onto $\Sigma$ with $F(+\infty)=+\infty$ and $F(-\infty)=-\infty$, extended to a homeomorphism of $\bar{S}$ with $\bar{\Sigma}$. Let $K$ be a constant and let $\left\{w_{n}=u_{n}+i v_{n}\right\} \subset \bar{S}$ be such that $u_{n} \rightarrow \infty$ and, for $z_{n}=x_{n}+i y_{n}=F\left(w_{n}\right)$.

$$
u_{n+1}-u_{n} \leq\left(x_{n}-u_{n}\right)+K \rightarrow+\infty \text {. }
$$

Then for $\operatorname{Re} F(w)=x(w), w \in \bar{S}$,

$$
x(w)-u \rightarrow+\infty \text { as } w=u+i v \rightarrow+\infty .
$$

Proof. Given $w=u+i v$, let $u_{n}$ be the largest of the $u_{n}$ for which $u-u_{n} \geq 2 M+2 \pi$. Let $u^{\prime}=u_{n}+M$ and $u^{\prime \prime}=u-M$. Then

$$
u^{\prime \prime}-u^{\prime}=(u-M)-\left(u_{n}+M\right)=\left(u_{n+1}-u_{n}\right)+\left(u-u_{n+1}\right)-2 M
$$

so that

$$
2 \pi \leq u^{\prime \prime}-u^{\prime}<u_{n+1}-u_{n}+2 \pi
$$

We define $\underline{x}_{u^{\prime \prime}}=\min \left\{x(w): w \in \sigma_{u^{\prime \prime}}\right\}$ and $\bar{x}_{u^{\prime}}=\max \left\{x(w): w \in \sigma_{u^{\prime}}\right\}$. By (3.2) and (3.3), $x_{n}<\bar{x}_{u^{\prime}}$ and $\underline{x}_{u^{\prime \prime}}<x$. Further, by (3.4) and (3.7)

$$
\int_{u^{\prime}}^{u^{\prime \prime}} d t / \theta(t)>1
$$

so that we may apply the Ahlfors distortion theorem (see p. 349 of [10]) to see that

$$
\begin{aligned}
x(w)-u & =x_{n}-u_{n}+\left(x-x_{n}\right)-\left(u-u_{n}\right) \\
& \geq x_{n}-u_{n}+\left(\underline{x}_{u^{\prime \prime}}-\bar{x}_{u^{\prime}}\right)-\left(u^{\prime \prime}-u^{\prime}\right)+\left(u^{\prime \prime}-u\right)+\left(u_{n}-u^{\prime}\right) \\
& \geq x_{n}-u_{n}+\int_{u^{\prime}}^{u^{\prime \prime}}((\pi-\theta(t)) / \theta(t)) d t-2 \pi-2 M \\
& \geq x_{n}-u_{n}-\left(\frac{1}{2}\right)\left(u^{\prime \prime}-u^{\prime}\right)-2 \pi-2 M \\
& \geq x_{n}-u_{n}-\left(\frac{1}{2}\right)\left(u_{n+1}-u_{n}\right)-3 \pi-2 M \\
& \geq x_{n}-u_{n}-\left(\frac{1}{2}\right)\left(x_{n}-u_{n}\right)-3 \pi-2 M-K / 2 \rightarrow \infty
\end{aligned}
$$

as $n \rightarrow \infty$. The last inequalities followed from (3.7) and (3.5). 
Remark. The above method may be applied to infer that the quotient $|\Psi(z)-1| /|z-1|^{\beta}$ is bounded or converges to 0 for an unrestricted approach of $z$ to 1 in $\bar{D}^{*}$ from hypotheses about the approach on "relatively thick" sequences. We have proved only the result of immediate interest. For any $\beta \in(0,2]$, the above method can be used to prove the following corollary, which applies to the conformal mapping of either the exterior or interior of $C$ onto the exterior or interior of the circle. We state it for the case at hand.

Corollary 3. Suppose that $C=\partial D$ is a quasicircle and that $\Psi$ maps $D^{*}=\operatorname{ext} C$ onto $\Delta^{*}=\{|w|>1\}$. Suppose that there is an arc $\gamma \subset \bar{D}^{*}$ with one endpoint at $z=1$, such that as $z \rightarrow 1$ along $\gamma, \Psi(z) \rightarrow 1$, and the quotient $|\Psi(z)-1| /|z-1| \rightarrow 0$ (or is bounded), then for an unrestricted approach of $z$ to 1 in $\bar{D}^{*}$, the quotient $\rightarrow 0$ (or is bounded).

We now use the method of strip mappings to construct a domain with smooth boundary and of bounded boundary rotation, symmetric with respect to the real axis, for which the conformal mapping $\Phi$ from $\Delta^{*}$ onto $D^{*}$ is locally Lip(1) at each $w \in \partial \Delta^{*}$ except $w=1$, and, at $w=1$, the difference quotient grows at a prespecified slow rate. In the following we will write $a(x) \approx b(x)$ to mean that the quotient $a(x) / b(x) \rightarrow 1$ as $x \rightarrow 0$.

Theorem 6. Fix a positive integer $k$ and let $L(x)=\log _{k}(x)=$ $\log (\log (\cdots(\log (x)) \cdots))$, the $k$-fold iterated logarithm. Then there exists a domain $D$, symmetric with respect to the real axis and with smooth boundary and of bounded boundary rotation, for which the conformal mapping function $\Phi: \Delta^{*} \rightarrow D^{*}$ is locally Lip(1) at all $w \in \partial \Delta$, except for $w=1$, and such that

$$
|w-1| /|\Phi(w)-1| \approx L(1 /|w-1|) .
$$

Consequently, if $P_{n}$ is a polynomial of degree $n$ such that $\left\|P_{n}\right\| \leq 1$, then for some constant $A$, depending only on $L$, and for $n \geq L^{-1}(1)$

$$
\left\|P_{n}^{\prime}\right\| \leq A n L(n) \text {. }
$$

Proof. We define a strip $S=\left\{u+i v:-\infty<u<\infty, \varphi_{-}(u)<v<\varphi_{+}(u)\right\}$, where $\varphi_{+}(u)=\pi / 2$ for $u \leq 0, \varphi_{+}(u)=(\pi / 2)(1+(d / d u) L(u))$ for $u \geq L^{-1}(1)=u_{1}$, and $\varphi_{+}(u)$ is defined for $0<u<u_{1}$ so that $\varphi_{+}$is $C^{2}$ on $(-\infty, \infty)$ and

$$
\int_{-\infty}^{\infty}\left(\varphi_{+}^{\prime}(u)\right)^{2} d u=K_{1}<\infty .
$$

Define $\varphi_{-}(u)=-\varphi_{+}(u)$, and $\theta(u)=\varphi_{+}(u)-\varphi_{-}(u)$. Next let

$$
\Sigma=\{x+i y:-\infty<x<\infty,|y|<\pi / 2\}
$$

and let $F(w)=z(w)$ map $S$ conformally onto $\Sigma$ with $F( \pm \infty)=F( \pm \infty)$ and 
$F(0)=0$. Let $F^{-1}(z)=w(z)$. Then, by a theorem of Warschawski [16], there exists a constant $K_{2}$ such that for $w=u+i v$, as $u \rightarrow+\infty$,

$$
\begin{aligned}
u-x(w) & =\int_{u_{1}}^{u}((\theta(t)-\pi) / \theta(t)) d t+K_{2}+o(1) \\
& =\int_{u_{1}}^{u}\left(\pi L^{\prime}(t) / \theta(t)\right) d t+K_{2}+o(1) \\
& =L(u)+o(L(u)) .
\end{aligned}
$$

On account of the slow growth of $L$, we then have, for $z=x+i y$,

$$
u(z)-x=L(x)+o(L(x)) \text {. }
$$

We now let $z(\zeta)=\log ((\zeta+1) /(\zeta-1))$ and $\omega(w)=\left(1+e^{-w}\right) /\left(1-e^{-w}\right)$, so that the mapping $\omega(w(z(\zeta)))$ maps $\Delta^{*}$ conformally onto a domain $D^{*}$ with the properties stated above. Further, (3.10) implies that

$$
|(\zeta-1) /(\omega-1)| \approx L(1 /|\zeta-1|) .
$$

Changing $\zeta$ to $w$ and $\omega$ to $z=\Phi(w)$, we obtain (3.8). To prove (3.9) we observe that $z=1$ is the worst point for the behavior of $\Psi=\Phi^{-1}$, so that if we consider any $z_{0} \in \partial \Delta$ and $w_{0}=\Psi\left(z_{0}\right)$, we then know that, for some constant $K_{3}$,

$$
\left|\left(\Psi(z)-w_{0}\right) /\left(z-z_{0}\right)\right| \leq K_{3} L\left(1 /\left|z-z_{0}\right|\right) .
$$

We may then assume that $z_{0}=w_{0}=1$ and refer to the proof of Theorem 1 . For any polynomial $P_{n}$ of degree $n \geq L^{-1}(1)$, with $\left\|P_{n}\right\| \leq 1$ on $\bar{D}$, we have, for $|1-z|=\rho=1 / n L(n)$,

$$
\begin{aligned}
\left|P_{n}(z)\right| & \leq\left(1+K_{3} \rho L(1 / \rho) n / n\right)^{n} \\
& \leq\left(1+K_{3} L(n L(n)) /(L(n) n)\right)^{n} \\
& \leq \exp \left(K_{4}\right),
\end{aligned}
$$

since $L(n L(n)) / L(n) \rightarrow 1$ as $n \rightarrow \infty$. The proof of Theorem 1 then yields (3.9).

No importance attaches to the special form of the functions $L(n)$ in Theorem 6 , beyond the fact that they can be of "arbitrarily" slow growth. What is important in Theorem 7 in the next section are the relations (4.6) and (4.7); any function $L(z)$ with real coefficients for which these hold will suffice for our purposes.

\section{Lower Bounds for Derivatives of Polynomials}

We shall now consider the domain $D$ constructed above for which (3.8) holds and estimate $f_{n}^{\prime}(1)$, for $f_{n}$ the Faber polynomials for $D$. Note that the mapping function $\Phi(w)$ of $\Delta^{*}$ onto $D^{*}$ is real for $w$ real. Thus (3.8) holds without the absolute value signs, for $w$ real. Since $D$ is of bounded boundary rotation, the Faber polynomials for $D$ are uniformly bounded on $D$ [1, Theorem B]. 
Theorem 7. Let $D$ be the domain constructed above, for which

$$
\begin{aligned}
(w-1) /(\Phi(w)-1) & =\rho(w) \approx \log _{k}(1 /(w-1)) \\
& =L(1 /(w-1)) \text { as } w \rightarrow 1^{+} .
\end{aligned}
$$

Then there exists a sequence $\{n(j)\}$ such that for the Faber polynomials

$$
f_{n(j)}^{\prime}(1) \geq n(j) L(n(j)) / 2 \text {. }
$$

Further, there exists a constant $\kappa>0$ such that if $0<1-x \leq \kappa /(n(j) L(n(j)))$, then

$$
f_{n(j)}(1)-f_{n(j)}(x) \geq(1-x) n(j) L(n(j)) / 4 .
$$

Proof. We first write

$$
\rho(w)=\sum_{0}^{\infty} a_{n} w^{-n}
$$

so that

$$
1 /(\Phi(w)-1)=\rho(w) /(w-1)=\sum_{1}^{\infty} k_{n} w^{-n},
$$

where $k_{n}=a_{0}+a_{1}+\cdots+a_{n-1}$. Differentiating the left and right sides of (4.4), we obtain

$$
\Phi^{\prime}(w) /(\Phi(w)-1)^{2}=\sum_{n=0}^{\infty}(n+1) k_{n+1} w^{-(n+2)} .
$$

Now the Faber polynomial of degree $n$ for $D$ is given for $z \in D$ by

$$
f_{n}(z)=(1 / 2 \pi i) \int_{|w|=R>1}\left(w^{n} \Phi^{\prime}(w) /(\Phi(w)-z)\right) d w
$$

so that

$$
\begin{aligned}
f_{n}^{\prime}(1) & =(1 / 2 \pi i) \int_{|w|=R}\left(w^{n} \Phi^{\prime}(w) /(\Phi(w)-1)^{2}\right) d w \\
& =(1 / 2 \pi i) \sum_{j=0}^{\infty}(j+1) k_{j+1} \int_{|w|=R} w^{-(j+2)} w^{n} d w \\
& =n k_{n} .
\end{aligned}
$$

This is true for any bounded simply connected domain.

Because of the symmetry of $D$, all of the $k_{n}$ are real, and $\Phi(w)$ is real when $w$ is real. Thus, summing over the nonnegative $k_{n}$, with $w>1$,

$$
1 /(\Phi(w)-1) \leq \Sigma^{\prime} k_{n} w^{-n}
$$

Fix an integer $N_{L} \geq L^{-1}(1)$. Then, for the sums involving the nonnegative $k_{n}$,

$$
1 /(\Phi(w)-1)-\sum_{n<N_{L}}^{\prime} k_{n} w^{-n} \leq \sum_{n \geq N_{L}}^{\prime} k_{n} w^{-n} \text {. }
$$


If every $k_{n}$ in the sum on the right were $<L(n) / 2$, then

$$
1 /(\Phi(w)-1)-\sum_{n<N_{L}}^{\prime} k_{n} w^{-n}<\left(\frac{1}{2}\right) \sum_{N_{L}}^{\infty} L(n) w^{-n}
$$

Now

$$
\sum_{N_{L}}^{N} L(n) \approx N L(N) \quad \text { as } \quad N \rightarrow \infty,
$$

so that by a classical theorem of Pringsheim [13],

$$
\sum_{N_{L}}^{\infty} L(n) w^{-n} \approx L(1 /(w-1)) /(w-1)
$$

But this leads to a contradiction to (4.1). By this argument there must in fact exist a subsequence $\left\{k_{n(j)}\right\}$ of $\left\{k_{n}\right\}$ for which $k_{n(j)} \geq\left(\frac{1}{2}\right) L(n(j))$. With (4.5) this yields (4.2).

Because of the symmetry of $D$, each $f_{n(j)}^{\prime}(x)$ is real, for real $x$. Thus, for $x<1$, applying Bernstein's inequality (3.9) for $D$ twice,

$$
\begin{aligned}
\left|f_{n(j)}^{\prime}(1)-f_{n(j)}^{\prime}(x)\right| & \leq(1-x)\left\|f_{n(j)}^{\prime \prime}\right\| \\
& \leq(1-x) A^{2}(n(j) L(n(j)))^{2}\left\|f_{n(j)}\right\|^{2} .
\end{aligned}
$$

As remarked above, the Faber polynomials for $D$ are uniformly bounded on $D$, so we may choose $\kappa$, independent of $j$, for which $A^{2}\left\|f_{n(j)}\right\|^{2} \leq 1 /(4 \kappa)$. If $(1-x) \leq$ $\kappa /(n(j) L(n(j)))$, we then have

$$
\left|f_{n(j)}^{\prime}(1)-f_{n(j)}^{\prime}(x)\right| \leq n(j) L(n(j)) / 4
$$

from which we see that

$$
f_{n(j)}^{\prime}(x) \geq n(j) L(n(j)) / 4
$$

and (4.3) follows by integration.

Theorem 8. Let $D$ be a domain as constructed above, with $L(x)=\log _{k}(x)$, for positive integer $k$. Then, for any $\alpha \in(0,1)$, there exists $f$ analytic in $D$ and continuous in $\bar{D}$ with $E_{n}(f)=O\left(1 / n^{\alpha}\right)$ but for which $f$ is not in $\operatorname{Lip}(\alpha)$.

Proof. Fix any $\alpha \in(0,1)$ and let $\{n(j)\}, j=1,2,3, \ldots$, be the sequence from Theorem 7 for which (4.3) holds. Without loss of generality we may assume that, for a fixed $M>1,(n(j+1) / n(j))>M$. The constant $M$ will be chosen later. Define

$$
f(z)=\sum_{j=0}^{\infty}\left(f_{n(j)}(z)\right) / n(j)^{\alpha} .
$$

For each $N \geq 1$, let $v=v(N)$ be such that $n(v) \leq N \leq n(v+1)$. Then let

$$
u_{v}(z)=\sum_{j=0}^{0} f_{n(j)}(z) / n(j)^{\alpha} .
$$

The polynomial $u_{v}$ is of degree at most $N$, so that

$$
\left\|f-u_{v}\right\| \leq \sum_{j=v+1}^{\infty}\left\|f_{n(j)}\right\| / n(j)^{\alpha} \leq K_{1} / n(v+1)^{\alpha}<K_{1} / N^{\alpha},
$$


for an appropriate constant $K_{1}$, so that $E_{N}(f)=O\left(1 / N^{\alpha}\right)$. Now, to show that $f$ is not in $\operatorname{Lip}(\alpha)$, we choose $x_{n(j)}$ for which

$$
1-x_{n(j)}=\delta_{n(j)}=\kappa /(n(j) L(n(j)))
$$

for $\kappa$ as in Theorem 7. Then, noting that, for $0<1-x<1-\delta_{n(j)}$ and $r \leq j$, (4.3) implies that $f_{n(r)}(1)-f_{n(r)}(x)>0$, we see that

$$
\begin{aligned}
u_{n(j)}(1)-u_{n(j)}\left(x_{n(j)}\right) & =\sum_{r=0}^{j}\left(f_{n(r)}(1)-f_{n(r)}\left(x_{n(j)}\right)\right) / n(r)^{\alpha} \\
& \geq\left(f_{n(j)}(1)-f_{n(j)}\left(x_{n(j)}\right)\right) / n(j)^{\alpha} \\
& \geq\left(1-x_{n(j)}\right) n(j) L(n(j)) /\left(4 n(j)^{\alpha}\right) \\
& =\kappa / 4 n(j)^{\alpha},
\end{aligned}
$$

by (4.8). Next, observe that

$$
\begin{aligned}
\left|\left(f(1)-u_{n(j)}(1)\right)-\left(f\left(x_{n(j)}\right)-u_{n(j)}\left(x_{n(j)}\right)\right)\right| & \leq 2\left\|f-u_{n(j)}\right\| \\
& \leq 2 K_{1} / n(j+1)^{\alpha} \leq 2 K_{1} /\left(M^{\alpha} n(j)^{\alpha}\right) .
\end{aligned}
$$

Now choose $M$ to be such that $2 K_{1} / M^{\alpha} \leq \kappa / 8$, so that

$$
\left|f(1)-f\left(x_{n(j)}\right)\right| \geq \kappa /\left(8 n(j)^{\alpha}\right) .
$$

But $\delta_{n(j)}=\kappa /(n(j) L(n(j)))$, hence $1 / n(j)^{\alpha}=\left(\delta_{n(j)} L(n(j)) / \kappa\right)^{\alpha}$ and thus

$$
\left|f(1)-f\left(x_{n(j)}\right)\right| /\left|1-x_{n(j)}\right|^{\alpha} \geq \kappa^{(1-\alpha)} L(n(j))^{\alpha} / 8 \rightarrow \infty,
$$

as $n \rightarrow \infty$, and Theorem 8 is proved.

Acknowledgments. The research of A. Hinkkanen was partially supported by the U.S. National Science Foundation. The research of F. D. Lesley was done while visiting University College, London.

\section{References}

1. J. M. ANDERSON, J. Clunie (1985): Isomorphisms of the disc algebra and inverse Faber sets. Math. Z., 188:545-558.

2. J. M. Anderson, F. W. Gehring, A. Hinkkanen (1985): Polynomial approximation in quasidisks. In: Differential Geometry and Complex Analysis (I. Chavel, H. M. Farkas, eds.). Berlin, Heidelberg: Springer-Verlag, pp. 75-86.

3. V. V. ANDREEVSKI (1986): The geometric structure of regions, and direct theorems of the constructive theory of functions. Math. USSR-Sb., 54:39-56.

4. V. I. BELYI (1977): Conformal mappings and the approximation of analytic functions in domains with a quasiconformal boundary. Math. USSR-Sb., 31:289-317.

5. E. W. CHENEY (1966): Introduction to Approximation Theory. New York: McGraw-Hill.

6. D. GAIER (1987): Lectures on Complex Approximation. Boston: Birkhauser.

7. T. KÖVARI (1972): On the order of polynomial approximation by polynomials for closed Jordan domains. J. Approx. Theory, 5:362-373.

8. N. A. LEBEDEV, P. M. TAMRAZOV (1970): Inverse problems of approximation on regular compacta in the complex plane. Math. USSR-Izv., 4:1355-1405.

9. F. D. LESLEY (1976): Best approximation on smooth arcs. J. Approx. Theory, 18:378-382.

10. F. D. LESLEY (1982): Hölder continuity of conformal mappings at the boundary via the strip method. Indiana Univ. Math. J., 31:341-354. 
11. F. D. LeSLEY (1983): Domains with Lipschitz mapping functions. Ann. Acad. Sci. Fenn. Ser. A I Math., 8:219-234.

12. F. D. LESLEY (1985): Conformal mappings of domains satisfying a wedge condition. Proc. Amer. Math. Soc., 93:483-488.

13. A. Pringsheim (1904): Über den Divergenz-charakter gewisser Potenzreihen an der Convergenzgrenze. Acta Math., 28:1-30.

14. N. A. SHIRokov (1977): Approximate entropy of continua. Soviet Math. Dokl., 18:990-994.

15. G. SzEGÖ (1925): Über einen Satz von A. Markoff. Math. Z., 23:45-61.

16. S. E. WARSCHAWSKI (1942): On conformal mapping of infinite strips. Trans. Amer. Math. Soc., 51:280-335.

\section{J. M. Anderson}

Mathematics Department

University College

London WC1

England

A. Hinkkanen
Mathematics Department
University of Michigan
Ann Arbor
Michigan 48109-1003
U.S.A.

A. Hinkkanen

University of Michigan

Michigan 48109-1003

U.S.A.

\author{
F. D. Lesley \\ Department of \\ Mathematical Sciences \\ San Diego State University \\ San Diego \\ California 92182 \\ U.S.A.
}

